\title{
On the Design of Voltage-Controlled Sinusoidal Oscillators Using OTA's
}

\author{
ANGEL RODRÍGUEZ-VÁZQUEZ, MEMBER, IEEE, BERNABÉ LINARES-BARRANCO, \\ JOSÉ L. HUERTAS, MEMBER, IEEE, AND EDGAR SÁNCHEZ-SINENCIO, SENIOR MEMBER, IEEE
}

\begin{abstract}
A unified systematic approach to the design of voltage-controlled oscillators using only operational transconductance amplifiers (OTA's) and capacitors is discussed in this paper. Two classical oscillator models, i.e., quadrature and bandpass-based, are employed to generate several oscillator structures. They are very appropriate for silicon monolithic implementations. The resulting oscillation frequencies are proportional to the transconductance of the OTA and this makes the reported structures well-suited for building voltage controlled oscillators (VCO's). Amplitude stabilization circuits using both automatic gain control (AGC) mechanisms and limitation schemes are presented which are compatible with the transconductance amplifier capacitor oscillator (TACO). Experimental results from bipolar breadboard and CMOS IC prototypes are included showing good potential of OTA-based oscillators for high frequency VCO operation.
\end{abstract}

\section{INTRODUCTION}

$\mathrm{T}$ HE generation of sinewaves is a classical problem with application in communication systems, instrumentation, measurement, etc. In particular, the voltagecontrolled frequency-variable oscillator (VCO) has a number of important applications in communication circuits [1], [2].

A great variety of $R C$-active circuits have been developed for generating sinewaves, most of them based on the use of the conventional operational amplifier (op amp) as the active component ([3]-[6] and included references). These circuits typically perform correctly in the audio frequency range $(\leqslant 20 \mathrm{kHz})$, but their performance becomes severely degraded as the frequency increases. Even by either using special design techniques that exploit the opamp reactive behavior [7] or resorting to composite amplifiers [8], [9], the useful frequency range of $R C$-active oscillators is below $100 \mathrm{kHz}$ for general purpose 741 op amps.

Together with these frequency limitations, op amp-based VCO's exhibit other problems further restricting their use-

Manuscript received August 1, 1988; revised March 9, 1989. A Rodríquez-Vázquez and J. L. Huertas were supported by the Spanish CICYT under Contract ME87-0004. This paper was recommended by Associate Editor C. A. T. Salama.

A. Rodriquez-Vázquez and J. L. Huertas, and B. Linares-Barranco are with the Department of Electronics and Electromagnetics, Faculty of Physics, University of Seville, Seville 41012, Spain.

E. Sánchez-Sinencio is with the Department of Electrical Engineering, Texas A\&M University, College Station, TX 77843.

IEEE Log Number 8932874

In bipolar and weak inversion MOS OTA's $g_{m}=h_{B} I_{\mathrm{BIAS}}$ and $g_{m}=h_{M}$ $\sqrt{I_{\text {BIAS }}}$ for MOS OTA in the saturation region where $h_{B}$ and $h_{M}$ are dependent upon temperature, device geometry and the process. fulness. Different variable-frequency $R C$-active oscillators have been reported whose frequency of oscillation can be controlled by a single resistor without affecting the oscillation condition [ 6 and included references]. Some additional circuitry has to be added to achieve VCO operation using these oscillator structures. One possible method is obtained by substituting the controlling resistor by a FET working in the ohmic region [10]. This consequently reduces the voltage swing across the simulated resistance. Besides, the tunable frequency range is somewhat reduced and switching among different resistors is needed what makes the design approach not readily compatible with monolithic integrated circuits.

Most of these problems can be overcome by the use of the operational transconductance amplifier (OTA) as the active building block for VCO's. By interconnecting OTA's and capacitors (TAC), oscillating circuits can be obtained whose frequency of oscillation is proportional to the transconductance gain $g_{m}$ of the OTA. Since the transconductance gain of the OTA can be varied or programmed by an external power supply, ${ }^{1}$ the VCO operation can be readily implemented. Thus fully integrated voltage-controlled TAC Oscillators (TACO's) can be obtained with a frequency adjustable over wide ranges, avoiding the necessity of switching among several passive components as observed in opamp designs.

TAC oscillator structures are also shown in this paper to be advantageous in terms of the maximum frequency of operation. As compared to the conventional opamp, transconductance amplifiers exhibit a higher potential for high-frequency applications [11]. This is an inherent property due to the fact that no internal high-impedance nodes are included in a typical transconductance amplifier architecture. As a matter of fact, several TAC filter structures have been recently reported for operation at frequencies in the range of megahertz [12]-[14]. Here we show that by using TACO's it is possible to push the oscillation frequency well above the maximum opamp oscillator ratings.

In this paper we present several TACO structures and discuss them by taking into account both linear and nonlinear effects. The herein reported TACO's are generated from two classical oscillator models, namely the quadrature and the bandpass oscillators. In order to make the paper self-contained, we first discuss the quadrature and the bandpass models in some detail in Section II. Section

0098-4094/90/0200-0198\$01.00 (C)1990 IEEE 
III is devoted to the generation of TACO's from the oscillator models. Section IV addresses the topics of amplitude control and distortion. Section V considers OTA parasitics and Section VI presents some experimental results. Most of the material in this paper can be used for either discrete bipolar or monolithic CMOS implementations. Experimental results covering these two cases are included. These results shows very high potential of TACO's for VCO high-frequency applications.

\section{The QuAdrature AND BandPass-Based OSCILLATOR}

\section{A. The Quadrature Oscillator Model}

An ideal quadrature oscillator consists of two lossless integrators (one inverting and one non-inverting) cascaded in a loop, what results in a characteristic equation with a pair of roots lying on the imaginary axis of the complex frequency plane. In practice, however, parasitics may cause the roots to be inside the left half of the complex frequency plane, hence avoiding the oscillation to begin. Any practical oscillator must include some form of regeneration to ensure that the roots are initially located in the right half plane and hence that the oscillation is created [1]. Besides, some amplitude stabilization mechanism must be added to pull the roots back towards the imaginary axis until a stable value for the amplitude is obtained.

A practical quadrature model including an amplitude stabilization scheme of the limiting type [1] is shown in Fig. 1, where $f(\cdot)$ represents a weakly nonlinear limiter. Analysis applying the chain differentiation rule gives, for Fig. 1:

$$
\frac{d^{2} x}{d t^{2}}-\frac{d x}{d t}\left[b_{1} \frac{d f(x)}{d x}-b_{2}\right]+\Omega_{0}^{2} x=0
$$

where $b_{1}, b_{2}$, and $\Omega_{0} \doteq\left(\omega_{01} \omega_{02}\right)^{1 / 2}$ are positive numbers. The following equations have to be fulfilled for the existence and uniqueness of stable quasi-sinusoidal oscillation in the weakly nonlinear system of Fig. 1 [15]:

$$
\begin{gathered}
\left.b_{1} \frac{d f(x)}{d x}\right|_{x=0}-b_{2}>0 \\
\left.b_{1} \frac{d f(x)}{d x}\right|_{x=x_{0}}-b_{2}<0, \text { for some } x_{0}>0
\end{gathered}
$$

where $f(x)$ is assumed to be odd-symmetric and $d f / d x$ is assumed to be decreasing for $x>0 .^{2}$

Imposing the condition in (2a) means that the roots of the characteristic equation of the linearized model of Fig. 2 are initially inside the right-half plane; it ensures that the system is self-starting. On the other hand, (2b) means that these roots move towards the left-half plane once the oscillation amplitude increases.

\footnotetext{
${ }^{2}$ Buonomo et al. give conditions for an oscillator composed by a Buonomo et al. give conditions for an oscillator composed by a
nonlinear element and a passive linear network. Although in this paper the linear network is active, the conditions are still valid providing the oscillator is represented by the block-diagram of Fig. 1. Also, we assume the nonlinear function $f(\cdot)$ is odd-symmetric for simplicity, but this condition is not required in the paper by Buonomo et al.
}

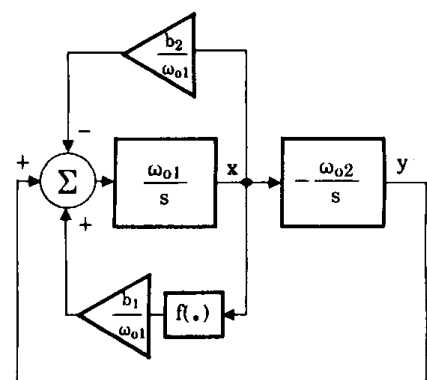

Fig. 1. Block diagram for a practical quadrature oscillator including a

The oscillation frequency and amplitude can be calculated by using the describing function approach [16]. The input of the nonlinearity is assumed to be an undistorted sinusoidal signal, $x(t)=A \sin (\omega t)$. The output of the nonlinear block is first expanded into a Fourier series and then this series is truncated to include the fundamental harmonic component. The describing function ${ }^{3}$ is the gain relating the amplitude of this fundamental component to that of the input,

$$
N(A)=\frac{\omega}{n A} \int_{0}^{2 n / \omega} f[A \sin (\omega t)] \sin (\omega t) d t .
$$

The following characteristic equation results for Fig. 1 by using the describing function:

$$
s^{2}-s\left[b_{1} N(A)-b_{2}\right]+\Omega_{0}^{2}=0 \text {. }
$$

The oscillation frequency $\omega_{0}$ and amplitude $A_{0}$ can be obtained by first making $s=j \omega$ and then considering separately the real and imaginary parts of (4). The resulting limit cycle is stable provided that the coefficient of the $s$-term in (4) is positive when $A>A_{0}$ and negative otherwise. It is fulfilled in case the nonlinear function $f(\cdot)$ has a decreasing derivative.

All the previous discussions can be summarized in the following design equations:

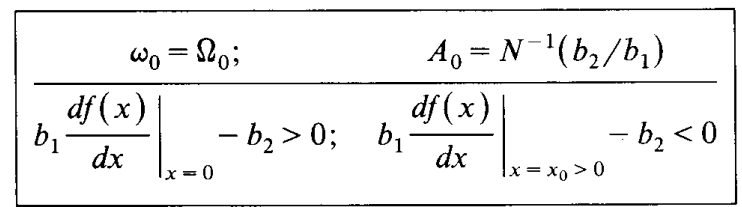

where an odd-symmetric nonlinearity have been assumed. The upper equations give a first-order approximation of the frequency and amplitude, while the lower ones ensure that oscillation exists, is stable, and unique.

In Fig. 1 the amplitude is controlled by a limiting function. Automatic gain control (AGC) is a more sophisticated scheme which usually reduces distortion [1]. Fig. 2 shows the block diagram for an AGC stabilized quadrature oscillator. Note that the block for $f(\cdot)$ is no longer present.

${ }^{3}$ Obviously an error results as a consequence of the use of the describing function. For quasi-sinusoidal oscillators this error is expected to be small since low-distortion signals are produced. A method for the error analysis of the describing function approach can be read from [17]. 


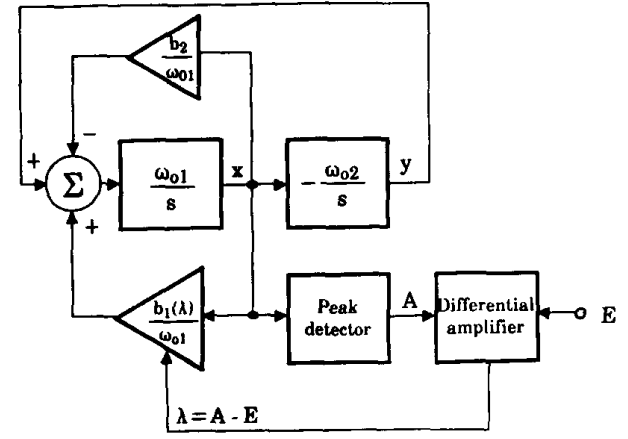

Fig. 2. Block diagram for a practical quadrature oscillator including an AGC mechanism.

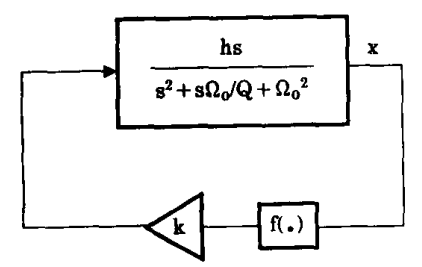

(a)

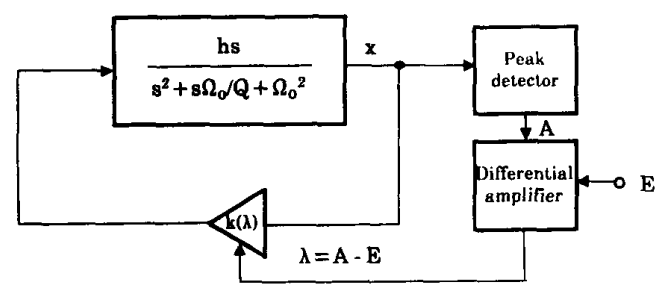

(b)

Fig. 3. The Bandpass-based oscillator model. (a) With a limiting type stabilization scheme. (b) With an AGC scheme.

Observe in Fig. 2 that the amount of positive feedback around the noninverting integrator depends on the difference, denoted by $\lambda$, between the oscillator output amplitude, $A$, and an external reference level, $E$. In steady-state both the positive and the negative feedback loops are linear and the system can be described by the following equation;

$$
\frac{d^{2} x}{d t^{2}}-\frac{d x}{d t}\left[b_{1}(A-E)-b_{2}\right]+\Omega_{0}^{2} x=0
$$

where $b_{1}(\cdot)$ is used to denote a functional dependence. The design equations for this case are

$$
\begin{array}{|cc|}
\hline \omega_{0}=\Omega_{0} ; & A_{0}=E+b_{1}^{-1}\left(b_{2}\right) \\
\hline\left.b_{1}(A-E)\right|_{A=0}-b_{2}>0 ; & \left.\frac{d b_{1}}{d A}\right|_{A=A_{0}}<0 . \\
\hline
\end{array}
$$
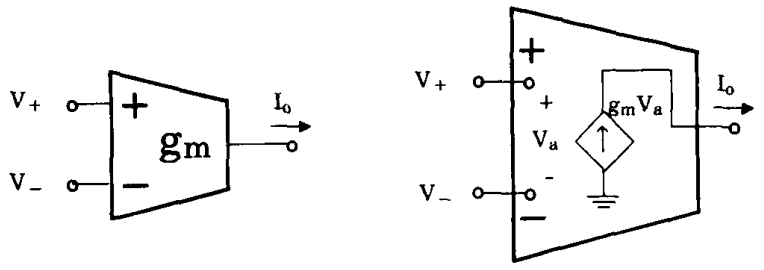

Fig. 4. Symbolic and ideal model for the OTA

\section{B. Bandpass-Based Oscillator Model}

The bandpass-based oscillator consists of a bandpass filter structure with positive feedback. Fig. 3(a) shows the block diagram for a bandpass oscillator including a stabilization mechanism of the limiting type. Fig. 3(b) shows the corresponding block diagram for a general AGC mechanism.

Analysis allows us to derive the following differential equation from Fig. 3(a):

$$
\frac{d^{2} x}{d t^{2}}-\frac{d x}{d t}\left[k h \frac{d f(x)}{d x}-\frac{\Omega_{0}}{Q}\right]+\Omega_{0}^{2} x=0
$$

which corresponds to (1) by defining $k h \doteq b_{1}$ and $\Omega_{0} / Q \doteq$ $b_{2}$. In a similar way, the characteristic equation for Fig. 3(b) can be obtained from (6) after making $b_{1}(\lambda)=h k(\lambda)$ and $b_{2}=\Omega_{0} / Q$. Equation (5) is thus valid for designing either quadrature or bandpass-based oscillators including a limiting type scheme, while (7) applies either for quadrature or bandpass AGC oscillators.

There is a particularly simple implementation of the limiting-type bandpass oscillator concept where the nonlinearity is of the ON-OFF type. The corresponding block diagram is readily obtained from Fig. 3(a) by substituting the cascade of the amplifier and nonlinear block by a comparator. The existence of oscillations for this system is a consequence of the very high gain of the comparator and thus does not require any other design conditions to be fulfilled [16]. Besides, the local stability of the resulting limit cycle is guaranteed because $d N(A) / d A<0$ for an $\mathrm{ON}-\mathrm{OFF}$ nonlinearity. The oscillation frequency is also given by (5). However, since now it is $b_{1}=h k \gg 1$, the amplitude is not conveniently expressed using this equation but as $A_{0}=4 E_{s} h Q / \pi \Omega_{0}[16]$, where $E_{s}$ denotes the comparator saturation levels.

This concludes this review section on oscillator models. Next sections of the paper address the problem of implementing previous models by using TAC structures.

\section{Generation of TAC Oscillator STRUCTURES}

Until otherwise stated, we will use the ideal OTA model of Fig. 4. The transconductance gain of the OTA is assumed to be a positive number. Interchanging the OTA input terminals is equivalent to substitute $g_{m}$ by $-g_{m}$.

The systematic application of the OTA for different useful linear functions can be read elsewhere [18]-[20]. As can be seen from Fig. 1 the basic building blocks for 


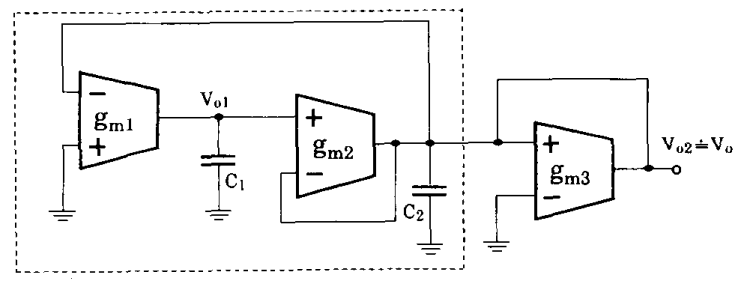

(a)

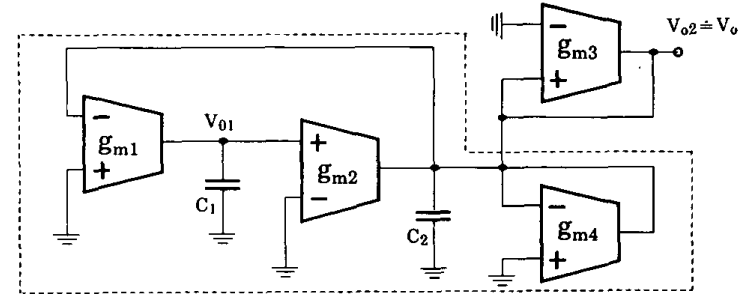

(b)

Fig. 5. TAC quadrature oscillator structures.

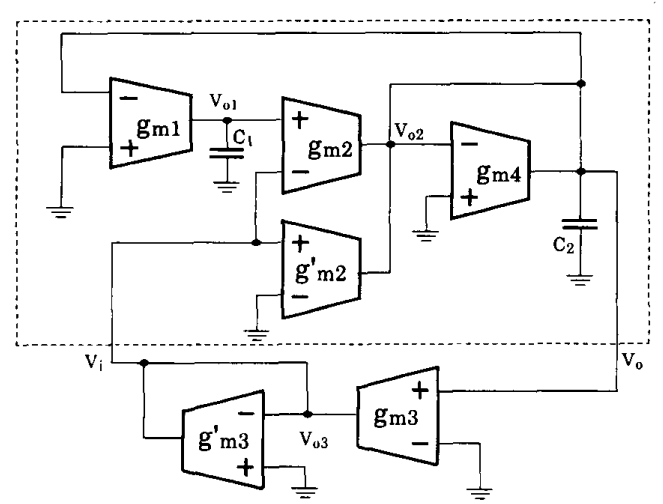

(a)

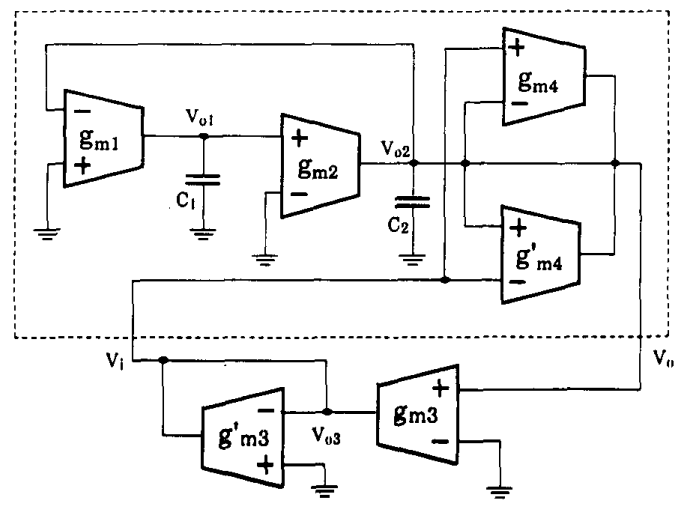

(b)

Fig. 6. TAC bandpass oscillator structures.

TABLE I

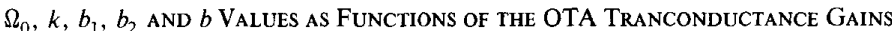
FOR THE DifFERENT OSCILlator STRUCTURES

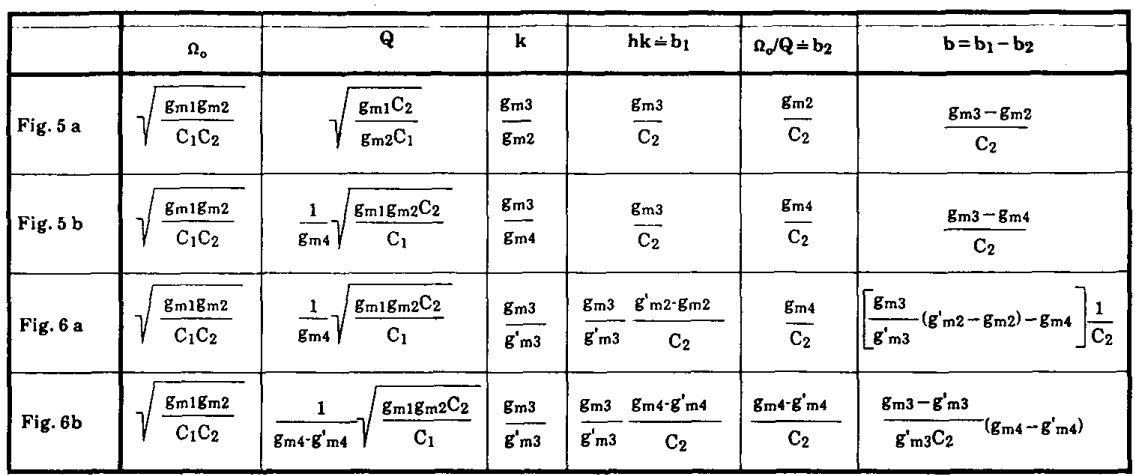

quadrature oscillators are integrators. In a similar way, state-variable realizations of second-order bandpass filters also use TAC integrators as the basic building blocks [19].

\section{A. TAC Oscillator Structures}

Different TACO structures are given in this subsection and analyzed using linear models. The control of the amplitude in these structures will be addressed in Section IV.

Fig. 5 shows two quadrature TACOS. They have been obtained from Fig. 1 by using TAC lossless and lossy integrators and OTA-simulated linear resistors [20]. Both circuits in Fig. 5 can be described by a second-order characteristic equation:

$$
\left(s^{2}-b s+\Omega_{0}^{2}\right) V_{0}(s)=0 .
$$

The first two rows of the first column in Table I give the expression of $\Omega_{0}$ as a function of the transconductance gains for these quadrature TACO's. The corresponding rows of the sixth column give the expression for $b$. We shall postpone any further comment on quadrature TACO design until the next section. 


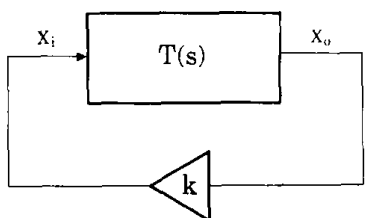

$k T(s)=1$

Fig. 7. Basic single feedback oscillator model and characteristic equation.

Fig. 6 shows two bandpass-TACO alternatives including the bandpass filter (enclosed by broken lines) and the voltage amplifier. The bandpass filter structures in Fig. 6 have been selected because they 1) exhibit high-input impedances, 2) allow us to independently tune the filter gain, the quality factor an the resonant frequency. These features give high flexibility in oscillator design [19], [21]. ${ }^{4}$ Looking at the first and sixth columns of Table I the third and fourth row give the expressions for $\Omega_{0}$ and $b$ of these bandpass-TACO's.

\section{B. A Unified Feedback Model for TAC Quadrature and Bandpass Oscillators}

Most of the existing theory for sinusoidal oscillator analysis (see [1], [15], [22] as some significant references) is based on modeling the oscillator structure by the basic feedback loop in Fig. 7, where the characteristic equation of the model has been included.

The bandpass TACO's of Fig. 6 are directly modeled by Fig. 7, $T(s)$ being the non-inverting transfer function of the bandpass filter and $k=g_{m 3} / g_{m 3}^{\prime}$. Analysis of Fig. $6($ a) gives

$$
T(s)=\frac{s\left(g_{m 2}^{\prime}-g_{m 2}\right) / C_{2}}{s^{2}+s g_{m 4} / C_{2}+g_{m 1} g_{m 2} / C_{1} C_{2}}
$$

while, for Fig. 6(b),

$$
T(s)=\frac{s\left(g_{m 4}-g_{m 4}^{\prime}\right) / C_{2}}{s^{2}+s\left(g_{m 4}-g_{m 4}^{\prime}\right) / C_{2}+g_{m 1} g_{m 2} / C_{1} C_{2}} .
$$

In order to obtain a similar feedback-loop model for quadrature TACO's, it is first convenient to redraw either Fig. 5(a) or (b) as illustrated in Fig. 8a, where the block labeled $Z(s)$ corresponds to the portion enclosed by broken lines in those figures. The corresponding feedback loop is then shown in Fig. 8(b), where, for convenience, the feedback signal has been normalized by $g_{m p}\left(g_{m p}\right.$ is defined in Fig. 8).

Using Fig. 8(a), the following transfer function results for the forward path of the feedback model corresponding to the quadrature oscillator of Fig. 5(a):

$$
T(s)=Z(s) g_{m 2}=\frac{s g_{m 2} / C_{2}}{s^{2}+s g_{m 2} / C_{2}+g_{m 1} g_{m 2} / C_{1} C_{2}}
$$

\footnotetext{
${ }^{4}$ The OTA $g_{m 2}^{\prime}$ of Fig. $6(a)$ is used to equalize the maximum voltage swings at $V_{01}$ and $V_{02}$ [19]. It could be eliminated in case equalization is not needed. In this case, Fig. 6 (a) should have to be modified by either interchanging the input terminals of the OTA's $g_{m 1}$ and $g_{m 2}$ or interchanging the input leads of the OTA $g_{m 3}$.
}

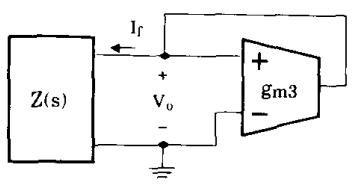

$Z(s)=V_{0}(s) / I(s)$

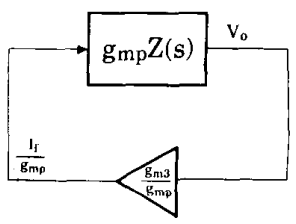

Fig. 8. Modeling quadrature TACO's by the interconnection of oneports $\left(g_{m p} \stackrel{\doteq}{=} g_{m 2}\right.$ for Fig. $5(\mathrm{a}), g_{m p} \stackrel{\doteq}{=} g_{m 4}$ for Fig. $5(\mathrm{~b})$.

being, for this structure, $k=g_{m 3} / g_{m 2}$. In the case of Fig. 5(b), the following $T(s)$ results:

$$
T(s)=Z(s) g_{m 4}=\frac{s g_{m 4} / C_{2}}{s^{2}+s g_{m 4} / C_{2}+g_{m 1} g_{m 2} / C_{1} C_{2}}
$$

with $k=g_{m 3} / g_{m 4}$.

In summary, the different TACO structures presented in the paper can be modeled by the feedback loop in Fig. 7, consisting of a forward path with a transfer function of the bandpass type,

$$
T(s)=\frac{s h}{s^{2}+s\left(\Omega_{0} / Q\right)+\Omega_{0}^{2}}
$$

and a feedback path described by a transconductance gain ratio $k$. This feedback model hence provides a unified framework for comparison among the different TACO's. Table I shows the expressions for the different relevant parameters associated to the feedback model as functions of the transconductance gains for Figs. 5 and 6.

\section{Some Observations on the Structures}

Design Considerations: Parameter $b$ controls both the oscillation condition and the amplitude of the oscillations. Similarly, the level of distortion can be also shown to depend on $b$. Note from Table I that the OTA transconductance gains appearing in the expressions for $\Omega_{0}$ of Figs. 5(b) and 6(b) are different to that appearing in the corresponding expressions for $b$. It means that we can tune the oscillation frequency by changing any of the transconductance gains that control $\omega_{0}$ and without affecting neither the oscillation condition nor the amplitude of the oscillation. This feature is very appropriate for VCO operation. On the contrary, for Figs. 5(a) and 6(a) the oscillation frequency and the oscillation condition are not independent, what makes these latter structures to be disadvantageous from the tuning point of view.

Observe from (10b) that the $T(s)$ gain at the center frequency is fixed to 1 for Fig. 6(b), while it is not constrained for Fig. 6(a). Since regeneration in the feedback loop must be guaranteed at the design stage, it means that the gain, $k$, of the feedback path is constrained to be greater than 1 for Fig. 6(b), while it can be set to 1 for Fig. 6(a). Hence, there is the possibility of substituting the two OTA's in the feedback path of Fig. 6(a) by a single wire. Note, on the other hand, that only 3 OTA's are required for Fig. 5(a) while 4 OTA's are required for Fig. 5(b). Summarizing, the structures of Figs. 5(a) and 6(a) are simpler than their counterparts in the sense that they 
require less active components, what makes them interesting for those applications where tuning is not a critical task.

Signal Scaling and Node Voltage Equalization: There is a limitation on the maximum OTA differential input voltage that ensures linear operation of the device. Besides, since in the proposed oscillator structures all the ungrounded OTA input leads are connected to an OTA output, voltage limitation is referred to both the input and the output of the OTA. Analysis of the maximum voltage swing at the different points inside the circuits is hence required for proper design of TACO's. Table II shows a set of expressions giving the relevant voltage amplitude ratios as measured at the frequency of oscillations for the different TACO structures. For convenience the node voltages $V_{01}$ and $V_{03}$ as well as the OTA differential input voltages $V_{a i}$ are referred to $V_{02}\left(V_{a i}\right.$ is the input to the OTA of transconductance $g_{m i}$ ); observe that by construction it is $\left|V_{a 1}\right| \equiv\left|V_{02}\right|$ for all the structures.

Different choices are possible when specifying the voltage amplitude ratios for a particular TACO design. The implementation of any particular election requires consideration of Tables I and II. Consider, for example, the TACO of Fig. 6(b) and note from Table I that the inequality $g_{m 4}>g_{m 4}^{\prime}$ must hold to ensure a positive quality factor. Assume we specify $\left|V_{03}\right|$ to be greater than $\left|V_{02}\right|$. From Table II it means $g_{m 3}>g_{m 3}^{\prime}$; this resulting in a negative value of $b$ from Table $\mathrm{I}$, the circuit would not oscillate. Different situations regarding this problem may arise from the proposed oscillator structures, and for the type of limiter or gain control used in each case.

A very interesting design specification concerning amplitude scaling is to equalize all node voltage swings at the frequency oscillation. Together with improving the dynamic range, equalization means the possibility of having multiple outputs with the same voltage level; in particular, two equal-amplitude $90^{\circ}$ phase-difference signals would be obtained for all the structures, corresponding to the $V_{01}$ and $V_{02}$ node voltages. As seen from Table II, the condition allowing us to equalize $V_{01}$ and $V_{02}$ for each one of the proposed TACOs is $C_{1 g_{m 2}}=C_{2 g_{m 1}}$. Observe from Table I that the quality factor of Fig. $5(\mathrm{a})$ is fixed to a value $Q=1$ as a consequence of imposing the equalization condition. It is a drawback that can result in an increased distortion level for this structure. On the contrary, the TACO's of Figs. 5(b), 6(a) and 6(b) can be designed for amplitude equalization and, at the same time, allow us to separately control the $b, \Omega_{0}$, and $Q$ values, which is a very appearing feature.

\section{Amplitude Stabilization and Control}

Using the nonlinear saturation characteristic of the OTA is the simplest form of amplitude stabilization for TACO's. The effect of this is equivalent, in a first order approximation, to decreasing the transconductance gain of the saturated OTA and thus allows us to control the value of $b$. We shall postpone the consideration of this possibility until Section V. Adding a nonlinear circuit which acts
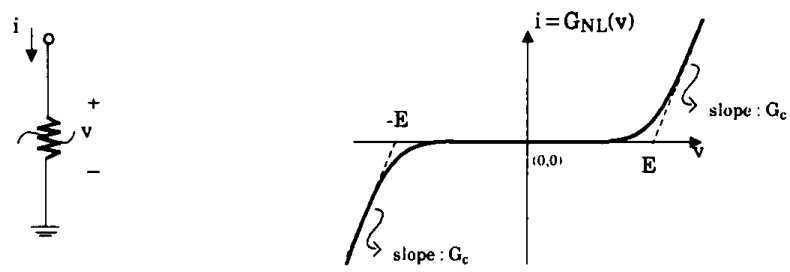

Fig. 9. Nonlinear resistor for amplitude limitation and control of TACO's.

TABLE II

Relevant voltage AMPLItUde Ratios for the Proposed TACO STRUCTURES

\begin{tabular}{|c|c|c|c|c|}
\hline & $\begin{array}{l}\text { Figure 5a } \\
\left|V_{a 1}\right|=\left|V_{o 2}\right| \\
\left|V_{\mathrm{a} 3}\right|=\left|V_{02}\right|\end{array}$ & $\begin{array}{l}\text { Figure 5b } \\
\left|V_{\mathrm{a} 1}\right|=\left|V_{o 2}\right| \\
\left|V_{a s}\right|=\left|V_{o 2}\right| \\
\left|V_{a 4}\right|=\left|V_{o 2}\right|\end{array}$ & $\begin{array}{c}\text { Figure 6a } \\
\left|V_{\mathrm{a} 1}\right|=\left|V_{02}\right| \\
\left|V_{\mathrm{a} s}\right|=\left|V_{02}\right| \\
\left|V_{\mathrm{a} 4}\right|=\left|V_{02}\right|\end{array}$ & $\begin{array}{l}\text { Figure } 6 b \\
\left|V_{\text {a1 }}\right|=\left|V_{o 2}\right| \\
\left|V_{\text {as }}\right|=\left|V_{02}\right|\end{array}$ \\
\hline$\frac{\left|V_{o 1}\right|}{\left|V_{02}\right|}$ & \multicolumn{4}{|c|}{$\sqrt{\frac{C_{2 g_{m 1}}}{C_{1 g_{m}}}}$} \\
\hline$\frac{\left|V_{03}\right|}{\left|V_{02}\right|}=\frac{\left|V_{{ }_{a 3}}\right|}{\left|V_{02}\right|}$ & \multicolumn{2}{|c|}{-} & \multicolumn{2}{|c|}{$\frac{g_{\mathrm{m} 3}}{\mathrm{~g}_{\mathrm{m} 3}}$} \\
\hline$\frac{\left|V_{\mathrm{a} 2}\right|}{\left|V_{02}\right|}$ & $\sqrt{1+\frac{C_{2 g_{m i}}}{C_{1} g_{m 2}}}$ & $\sqrt{\frac{\mathrm{C}_{2 \mathrm{gm}_{\mathrm{m}}}}{\mathrm{C}_{1 \mathrm{gm} 2}}}$ & $\sqrt{\frac{C_{2 \mathrm{gm} 1}}{C_{1 \mathrm{gm} 2}}+\left(\frac{\mathrm{g}_{\mathrm{m} 3}}{\mathrm{~g}_{\mathrm{m} 3}{ }^{\prime}}\right)^{2}}$ & $\sqrt{\frac{\mathrm{C}_{2 \mathrm{~g}_{\mathrm{m} 1}}}{\mathrm{C}_{1 \mathrm{~g}_{\mathrm{m} 2}}}}$ \\
\hline$\frac{\left|V_{\mathrm{a} 2}\right|}{\left|V_{\mathrm{o} 2}\right|}$ & \multicolumn{2}{|c|}{-} & $\frac{\mathrm{g}_{\mathrm{m} 3}}{\mathrm{~g}_{\mathrm{m} 3}^{\prime}}$ & - \\
\hline$\frac{\left|V_{\mathrm{a} 4}\right|}{\left|V_{\mathrm{o} 2}\right|}=\frac{\left|V_{\mathrm{a} 4}^{\prime}\right|}{\left|V_{\mathrm{o} 2}\right|}$ & \multicolumn{3}{|c|}{-} & $1-\frac{g_{m 3}}{g_{m}^{\prime} 3}$ \\
\hline
\end{tabular}

before the natural limitation of the OTA is a more sophisticated scheme providing better controllability. Finally, exploiting the programmability of the transconductance gain of the OTA is a natural and yet simple way to implement the AGC concept in TACO structures.

\section{A. Amplitude Control by External Limiters}

Limitations by Nonlinear Resistors: Three basic guidelines have to be followed when looking for limiting circuits for the proposed TACO's: 1) the operation of the limiter must be compatible with the OTA operation principle (voltage to current conversion), 2) the equations describing the global operation of the oscillator (TAC structure plus limiter) must be in accordance with the oscillator models in Section II, and 3) the implementation of the limiter must be possible for either the bipolar case or the CMOS case.A way to cope with all previous objectives is to use a nonlinear resistor described as in Fig. 9.5

Fig. 10(a) shows how to connect the nonlinear resistor to ensure proper amplitude control of quadrature TACO's, the block labeled $Z(s)$ being the port impedance of the circuit enclosed by broken lines in Fig. 5. Fig. 10(b) illustrates the corresponding connection for bandpass TACO's, $T(s)$ being the transfer function for the circuit enclosed by broken lines in Fig. 6 .

\footnotetext{
${ }^{5}$ In the discrete bipolar case this resistor can be implemented by using a linear resistor and a couple of diodes [23]. In the monolithic case the diodes can be substituted by MOS transistors [24] and the linear resistor simulated by OTA's.
} 


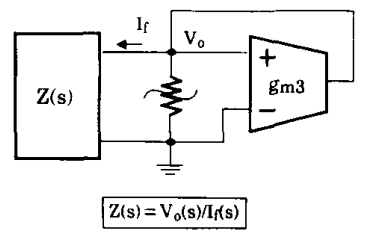

(a)

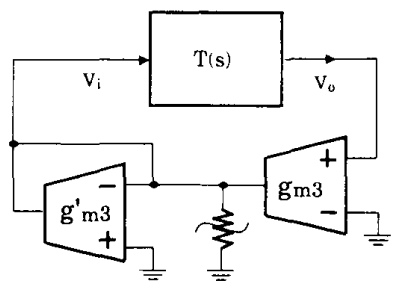

(b)
Fig. 10. Connection of the resistor for amplitude control of (a) quadrature TACO's and (b) bandpass TACOs.

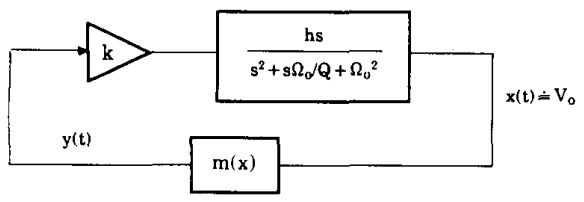

(a) (b)

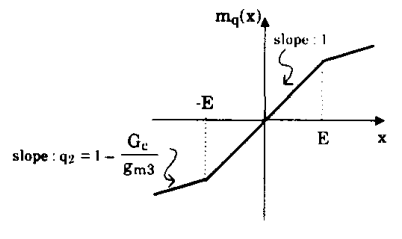

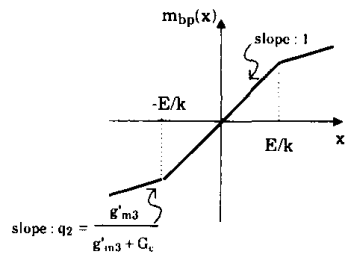

(c)
Fig. 11. (a) Unified nonlinear feedback loop model for TACO's. (b) Characteristics of the nonlinear block for quadrature TACO's. (c) Corresponding characteristics for bandpass TACO's.
We can now compare (12) with (1), keeping in mind the conditions given in (2). Note that $\left.\left(d m_{q} / d x\right)\right|_{x=0}=$ $\left.\left(d m_{b p} / d x\right)\right|_{x=0}=1$. Thus for amplitude-limited TACO's the following inequalities must hold for quasi-sinusoidal oscillations to exist and be unique:

$$
\begin{gathered}
b=h k-\left(\Omega_{0} / Q\right)>0 \\
b^{\prime}=q_{2} h k-\left(\Omega_{0} / Q\right)<0
\end{gathered}
$$

where $q_{2}$ is the slope of the outer segments in Fig. 11(b) and (c).

The frequency and the amplitude of the oscillations can be calculated using the describing-function approach:

$$
\begin{gathered}
\omega_{0}=\Omega_{0} \\
A_{0}=D N_{m}^{-1}\left(\Omega_{0} / Q h k\right)
\end{gathered}
$$

where $D$ denotes the value of the breakpoints of the characteristics of the nonlinear block of Fig. 11; being $D=E$ for quadrature TACO's (Fig. 11(b)) and $D=E / k$ for bandpass-based TACO's (Fig. 11(c)). On the other hand, $N_{m}(\cdot)$ is given by

$$
N_{m}(z)=\left(1-q_{2}\right) N_{1}(z)+q_{2}
$$

$z$ being an auxiliary variable and $N_{I}(\cdot)$ being

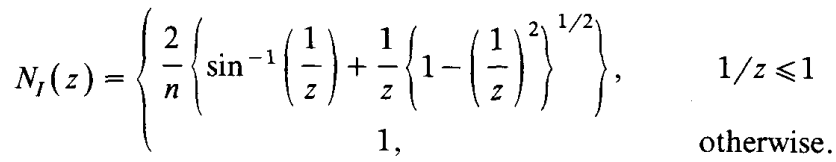

Fig. 11(a) shows a unified nonlinear feedback model valid either for quadrature or bandpass TACO's. The linear portion of this model is defined in Table $I$ for each particular TACO.

For quadrature TACO's the signal $y(t)$ in Fig. 11(a) is defined as $I_{f} / g_{m 3}$ where $I_{f}$ is the port current for the block $Z(s)$ as shown in Fig. 10(a). Since $I_{f}=g_{m 3} V_{0}-$ $G_{N L}\left(V_{0}\right)$, and taking, into account the definition of $G_{N L}(\cdot)$ in Fig. 9, the characteristic of Fig. 11(b) results in the nonlinear block in the case of quadrature TACO's $\left(G_{c}\right.$ in this figure is the slope of the external segment in Fig. 9). For bandpass TACO's, $y(t)=V_{i}(t) g_{m 3}^{\prime} / g_{m 3}, V_{i}(t)$ being defined in Fig. 10b, and the characteristic for the nonlinear block is the one shown in Fig. 11(c).

Analysis yields the following scalar differential equation for the unified nonlinear feedback TACO model:

$$
\frac{d^{2} V_{0}}{d t^{2}}-\frac{d V_{0}}{d t}\left\{h k \frac{d m\left(V_{0}\right)}{d v_{0}}-\frac{\Omega_{0}}{Q}\right\}+\Omega_{0}^{2} V_{0}=0
$$

that applies to quadrature TACO's (see (1)) by making $m(\cdot)=m_{q}(\cdot)$ and to bandpass TACO's (see (8)) for $m(\cdot)$ $=m_{p b}(\cdot)$.
Using (14b) together with Table I allows us to set the different OTA transconductance gains and capacitances for a given frequency and amplitude. For a given setting to correspond to a practical oscillator, we must also ensure that (14a) is fulfilled. Note that this latter equation constraints the sign of parameters $b$ and $b^{\prime}$ but does not impose any restriction on their values. Further insight on how to set these parameters can be gained by considering the distortion level at the output of the oscillators.

Distortion Analysis: Assume the distortion level for $x(t)$ in the model of Fig. 11(a) is small enough so that $y(t)$ can be expressed as the Fourier expansion of the output of the odd/symmetric nonlinear block when a pure sinusoidal signal, $A_{0} \sin \left(\omega_{0} t\right)$, is applied to its input:

$$
\begin{aligned}
y(t) & =\sum_{n=1}^{\infty} B_{n} \sin \left(n \omega_{0} t\right) \\
B_{n} & =\frac{\omega}{n} \int_{0}^{2 \pi / \omega} m\left\{A_{0} \sin \left(\omega_{0} t\right)\right\} \sin \left(n \omega_{0} t\right) d t .
\end{aligned}
$$

The different harmonic components of $x(t)$ can be calculated [1] by applying the superposition principle to the forward path in Fig. 11(a), the input signal being that 
in $(16 \mathrm{a})$,

$$
\begin{aligned}
x(t) & =\sum_{n=1}^{\infty} C_{n} \sin \left(n \omega_{0} t+\phi_{n}\right)=\sum_{n=1}^{\infty} H_{n} B_{n} \sin \left(n \omega_{0} t+\phi_{n}\right) \\
H_{n} & =\frac{h k}{\omega_{0}} \frac{n}{\left\{\left(n^{2}-1\right)^{2}+\frac{n^{2}}{Q^{2}}\right\}^{1 / 2}} \\
\Phi_{n} & =-\tan ^{-1}\left\{\frac{n / Q}{1-n^{2}}\right\}+\frac{n}{2} .
\end{aligned}
$$

Thus the following expression for the total harmonic distortion (THD) results:

$$
\begin{aligned}
\text { THD } & =100\left(\sum_{n=2}^{N} \frac{C_{n}^{2}}{C_{1}^{2}}\right)^{1 / 2} \\
& =100\left\{\sum_{n=2}^{N} \frac{\left(b_{1} / b_{2}\right)^{2}}{\left[1+Q^{2}\left(n-\frac{1}{n}\right)^{2}\right]^{2}} \frac{B_{n}^{2}}{C_{1}^{2}}\right\}^{1 / 2} .
\end{aligned}
$$

where $b_{1} \doteq h k, b_{2} \doteq \Omega_{0} / Q$, and $C_{1}$ is the amplitude for the fundamental harmonic, it is, $C_{1} \doteq A_{0}$, as calculated by (14b) using the describing function approach. On the other hand, $B_{n}$ can be easily calculated from (16b) to give

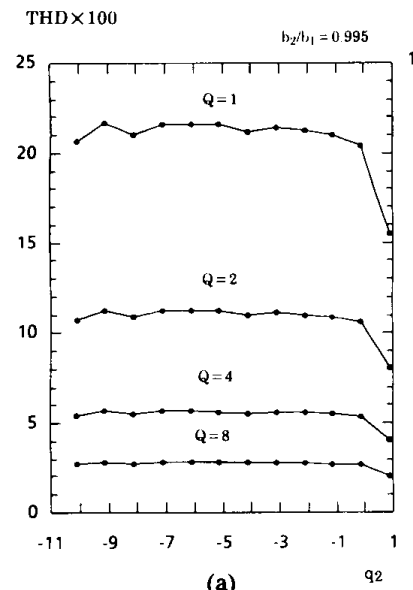

(a)

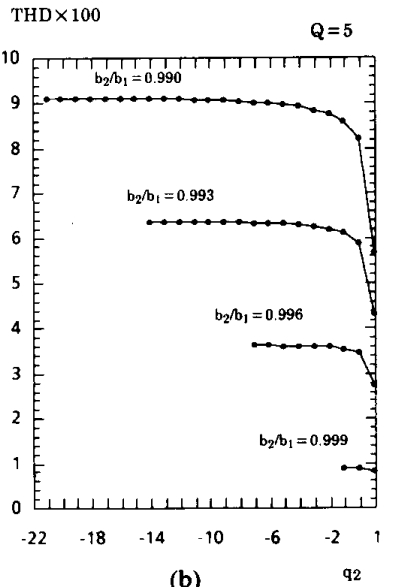

(b)

$\mathbf{9 2}$

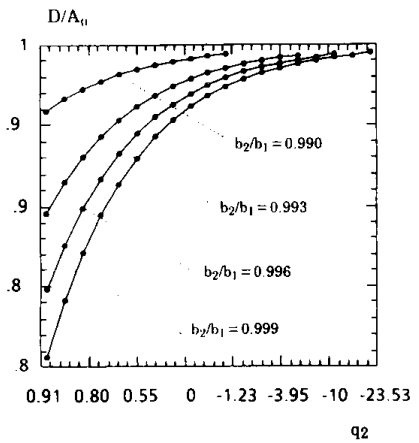

(c)
Fig. 12. (a), (b) Illustrating the influence of the design parameters on the THD. (c) Normalized oscillator amplitude versus $q_{2}$.

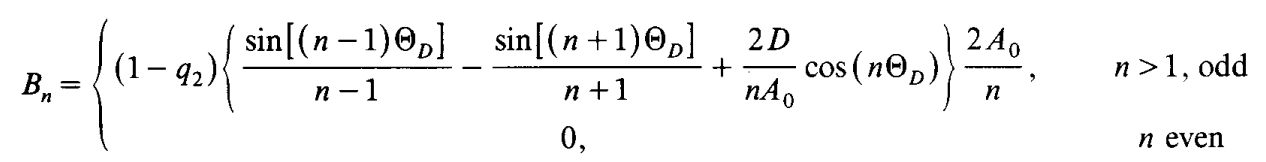

where we define $\Theta_{D} \doteq \sin ^{-1}\left(D / A_{0}\right), D$ representing the value of the breakpoint in Fig. 11(b) and (c). Observe from (19) that the actual value of the THD is a function of $b_{2} / b_{1}, Q$ and $q_{2}$. Fig. 12(a), (b) illustrate the dependence of THD on these parameters. Fig. 12(a) shows THD $\times 100$ versus $q_{2}$ (note from Fig. 11(b), (c) that $q_{2}$ is always smaller than 1) for different values of $Q$ and assuming $b_{2} / b_{1}=0.995$; similar families of curves result from different $b_{2} / b_{1}$ values. Fig. 12(b) shows THD $\times 100$ versus $q_{2}$ for different values of $b_{2} / b_{1}$ assuming $Q=5$.

We note the THD decreases as $Q$ increases and $b_{2} / b_{1}$ increases. On the other hand, the THD seems not to be much influenced by the values of $q_{2}$ with the exception of a sudden THD decrease when $q_{2}$ is near to 1 . We can exploit this for obtaining low values of the THD by using values of $q_{2}$ near the unity. However, a drawback appears in that it corresponds to large oscillator amplitudes and thus can drive the OTA's outside their linear input ranges. This can be seen from Fig. 12(c) where we represent $D / A_{0}$ as a function of $q_{2}$ for different $b_{2} / b_{1}$ values. As it can be seen, decreasing $q_{2}$ results in an increase of the ratio among the oscillation amplitude and the breakpoint $D$ of the characteristic of the nonlinear block.
The observations included in Section III-C on the different structures are confirmed by the above distortion analysis. Those structures allowing separate control of $b_{2} / b_{1}$, $\Omega_{0}$, and $Q$, as is the case for Figs. 5(b) and 6(b), are potentially advantageous as compared to the others. An additional observation concerns the different way parameter $q_{2}$ varies from either quadrature or bandpass-based TACO's. As it can be seen from the definition of $q_{2}$ in Fig. $11(\mathrm{~b})$ and (c) this parameter can be made to change inside the interval $(-\infty, 1]$ for quadrature TACO's, and inside $(0,1]$ for bandpass-based TACO's. Since the oscillator amplitude increases as $q_{2}$ increases above zero (see Fig. 12(c)), quadrature TACO's can be designed to exhibit smaller amplitude values than bandpass-based TACO's. A potential advantage of these former structures can be observed by taking into account that the amplitude must be maintained below an appropriate safety level to avoid OTA nonlinearities.

Amplitude Control by Voltage Comparators: Resorting to the unified feedback TACO model presented in Section III allows us to apply this technique to either bandpass or quadrature TACO's. Fig. 13(a) shows the block diagram for bandpass and Fig. 13(b) shows the corresponding 


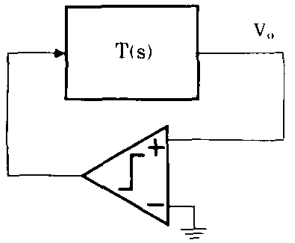

(a)

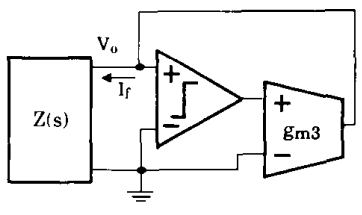

(b)
Fig. 13. Amplitude control using comparators. (a) Bandpass TACO. (b) Quadrature TACO

diagram for quadrature (see (10) and (11) for $T(s)$ and $Z(s)$, respectively).

Assume the saturation levels for the comparator for $\pm E_{s}$. The oscillation frequency and the fundamental harmonic amplitude of $V_{0}$ are, respectively [16]:

$$
\omega_{0}=\Omega_{0} ; A_{0}=\frac{4 E_{s}^{\prime} h Q}{n \Omega_{0}}
$$

where $E_{s}^{\prime}=E_{s}$ for bandpass TACO's and $E_{s}^{\prime}=k E_{s}$ for quadrature TACO's (see Table I for the expressions of $\Omega_{0}$, $h$ and $Q$ as functions of the transconductance gains). In steady state the feedback signal in Fig. 10 ( $I_{f}$ in Fig. 10(a) and $V_{i}$ in Fig. $10(\mathrm{~b})$ is a square wave. By first expanding it into a Fourier series and then computing the response of the linear portion of Fig. 13, we obtain the following relation for the $n$th harmonic distortion at the bandpass filter output,

$$
\frac{C_{n}}{C_{1}}=\frac{1}{Q} \frac{1}{\left(\left(1-n^{2}\right)^{2}+\frac{n^{2}}{Q^{2}}\right)^{1 / 2}}, \quad n \text { odd }
$$

where $C_{n}$ is the $n$th harmonic's amplitude and $C_{1} \doteq A_{0}, A_{0}$ being as calculated in (20). The very low input voltage range for linear operation of discrete bipolar OTA's discourages the use of this technique for discrete TACO's. However it can be very appealing in the monolithic case where the comparator can be implemented by using a simple CMOS inverter.

\section{B. Amplitude Control by $A G C$}

Exploiting the programmability of the transconductance gain of the OTA is a natural and yet simple way to implement the AGC concept in TACOs. Let us generically use $z$ to denote the programming variable of the OTA, $z$ being a bias current for discrete bipolar OTAs and a bias voltage for CMOS ones. Assume $z$ is allowed to vary $\Delta z$ around a quiescent value $z_{0}$. The following inequality holds for both types of OTA's:

$$
\frac{\Delta g_{m}\left(z_{0}+\Delta z\right)}{\Delta z}>0
$$

which means that he transconductance increases with $z$.

For any of the proposed TACO's, the AGC concept can be implemented by making the transconductance gain $g_{m 3}$

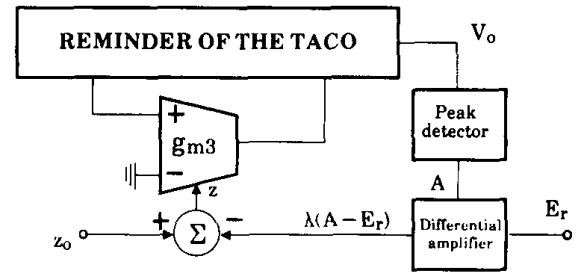

Fig. 14. AGC loop for amplitude control of TACO's.

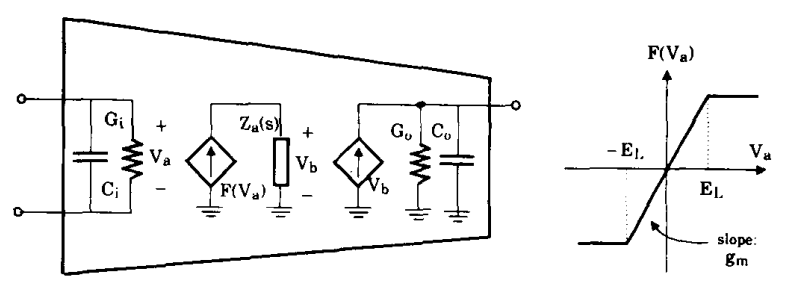

Fig. 15. Second-order OTA macromodel.

to depend on the difference between the peak amplitude of the oscillator output and an external reference $E_{r}$, as is illustrated in Fig. 14. Let us consider we want the oscillation amplitude to coincide wit the external reference level $E_{r}$. Note from Table I that $g_{m 3}$ controls parameter $k$ for all the proposed TACO's and that $k$ controls the value of parameter $b$. For the circuit oscillating with $A_{0}=E_{r}$, we require $b$ to be zero for such a value of the amplitude, i.e., the transconductance gains must be trimmed to fulfill the following equation:

$$
b\left(z_{0}\right)=k\left|g_{m 3}\left(z_{0}\right)\right| h-\left(\Omega_{0} / Q\right)=0 .
$$

Since $d k / d g_{m 3}>0$ and $\left.\left(d g_{m 3} / d A\right)\right|_{z_{0}}<0$ for Fig. 14, then $\left.(d k / d A)\right|_{A 0}<0$, which means that the roots of the characteristic equation return to the left half plane for the amplitude eventually increasing above $A_{0}$, and hence that oscillatory behavior is stable.

Different alternatives for AGC-TACO can be used involving control for one or more OTA's. For the bipolar case, where $z$ is a current, the required amplifier and the summing node can be implemented by an OTA and using Kirchoff's current law, respectively. Several possibilities for the monolithic case have been proposed by the authors and validated at the simulation level [21].

\section{OTA PARASITIC EFFEcts}

Second-order effects are specially important in oscillators, where even small parasitics can produce pole displacements large enough to cause the oscillation to vanish or to increase distortion severely. There are three main OTA parasitics affecting the performance of TACOs: 1) Finite input and output impedances, 2) frequency-dependent transconductance gain, and 3) output current saturation. Fig. 15 shows a macromodel including all of these parasitics.

Let us focus on the reactive behavior of the OTA modeled by $Z_{a}(s)$ in Fig. 15 . A dominant nonideal effect in the frequency response of the OTA is the phase error 
[21]. This is a phase lag that can be modeled by assuming a time delay $\tau \doteq 1 / \omega_{z}$ through each OTA [25]. It is, by making the transconductance to be frequency-dependent:

$$
g(s)=g_{m} \exp \left(-s / \omega_{z}\right)
$$

for $\omega \ll \omega_{z}$ we can approximate $g(s)$ by the two first terms of its Taylor expansion,

$$
g(s) \doteq g_{m}\left(1-s / \omega_{z}\right)
$$

thus a model consisting of a single zero on the right-half of the complex frequency plane is obtained. This model provides the same degree of approximation for the phase shift as the more conventional model consisting of a single left half plane pole [11]. However, it yields simpler expressions for the characteristic equation, a very appealing feature, allowing us to obtain by inspection useful information about the influence of second-order effects of TACO performance.

Let us, by the way of examples, illustrate how OTA parasitics influence the quadrature oscillator of Fig. 5(b). Very similar discussion and results apply to the other TACO structures herein.

OTA Linear Parasitics: Let us assume the different OTA's in Fig. 5(b) are working inside the linear region of
Two points are observed from this equation, which are worthy of consideration during the design stage of TACO's:

1) The roots of the characteristic equation are displaced from their nominal positions at $b / 2 \pm j\left(\Omega_{0}^{2}-\right.$ $\left.b^{2} / 4\right)^{1 / 2}$, because of parasitics. At low frequencies, where the effect of the OTA resistances are dominant, these roots move to the left and oscillation might vanish. At high frequencies, the effect of $\omega_{z}$ dominates and the roots move further into the righthalf plane, forcing the amplitude control mechanism to work harder and causing the distortion level to increase.

2) Parasitics cause the oscillation frequency and the coefficient of the $s$-term of the characteristic equation (parameter controlling the oscillation condition) to be interdependent, thus rendering the tuning process more involved than for the ideal case.

OTA Nonlinear Parasitics: Let us assume one or several of the OTAs in Fig. 5(b) are working outside the linear range of Fig. 15. Assume, anyway, that the different node voltages of Fig. 5(b) are sinusoidal signals,

$$
v_{0 i}=A_{0 i} \sin \left(\omega t+\delta_{i}\right), i=1,2 .
$$

The overall input-output behavior of each OTA [26] can be modeled in the frequency domain by using the describing function associated with the nonlinearity in Fig. 15. Thus the following approximate characteristic equation is obtained:

$$
s^{2}-s\left[\frac{\left(g_{m 3}-g_{m 4}\right) N_{1}\left(\frac{E_{L}}{A_{02}}\right)}{C}+2 \frac{g_{m 1} N_{1}\left(\frac{E_{L}}{A_{02}}\right) g_{m 2} N_{1}\left(\frac{E_{L}}{A_{01}}\right)}{C^{2} \omega_{z}}-4 \frac{G_{0}+G_{i}}{C}\right]+\frac{g_{m 1} N_{I}\left(\frac{E_{L}}{A_{02}}\right) g_{m 2} N_{1}\left(\frac{E_{L}}{A_{01}}\right)}{C^{2}}=0
$$

Fig. 15. Analysis gives the following characteristic equations:

$$
\begin{aligned}
s^{2}\{1 & \left.+\frac{\Omega_{0}^{2}}{\omega_{z}^{2}}+\frac{b}{\omega_{z}}\right\} \\
& -s\left\{b+2 \frac{\Omega_{0}^{2}}{\omega_{z}}-\frac{\left(G_{0}+G_{i}\right)}{C_{1}}\left(\frac{3 C_{1}}{C_{2}}+1+\frac{b}{\omega_{z}}\right)\right\} \\
& +\Omega_{0}^{2}\left\{1+\frac{\left(G_{0}+G_{i}\right)\left[3\left(G_{0}+G_{i}\right)+g_{m 4}-g_{m 3}\right]}{g_{m 1} g_{m 2}}\right\}=0
\end{aligned}
$$

where we have assumed that the OTA parasitic capacitors in Fig. 15 have been included in $C_{1}$ and $C_{2}$. As can be seen, parasitics result in a change in both the oscillator frequency and the oscillation condition. This latter change is however both qualitatively and quantitatively more significant. Let us take for convenience $C_{1}=C_{2}=C$ and consider $\omega_{z} \gg\left(\Omega_{0}, b\right)$ and $g_{m j} \gg\left(G_{0}+G_{i}\right), j=1,2,3,4$. Then (26) simplifies to

$$
s^{2}-s\left[b+2 \frac{\Omega_{0}^{2}}{\omega_{2}}-\frac{4\left(G_{0}+G_{i}\right)}{C}\right]+\Omega_{0}^{2}=0 .
$$

where the function $N_{I}(\cdot)$ is defined in $(15 b)$.

In practice, the effect of nonlinearities should be avoided through proper amplitude control and signal scaling. However, (28) suggests to use the OTA saturation characteristic for simple amplitude control of TACO's. For instance, assume $A_{01}>E_{L}, A_{02}<E_{L}$. Then $d g_{m 2} 2^{N L} / d A_{01}<0$ and it is possible to make the roots to move from the right-half plane into the left-half one when the frequency increases, as is required for obtaining a stable limit cycle oscillation.

\section{EXPERIMENTAL Results}

Several measurements on practical TACO's are presented in this section trying to 1) show the basic performance of the proposed circuits, 2) validate the OTA parasitic effects analysis, and 3) illustrate the use of TACO's for AGC and VCO. Most of the results are for breadboard bipolar prototypes. Besides, results from a CMOS monolithic prototype are included. For breadboard prototypes, commercial OTA's CA3080 and 10-percent tolerance capacitors $C_{1}=C_{2} \doteq C$ were used. Unless otherwise indicated, the OTA-CA3080 input leads are buffered by Darlington pairs CA3083 and resistive attenuators are included to achieve linear ranges of $200 \mathrm{mV}$ for the OTA differential input voltage. 

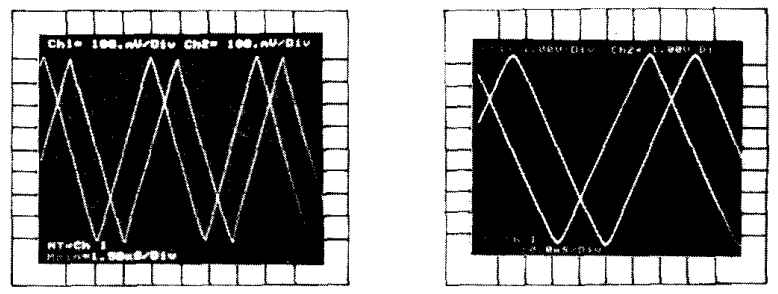

Fig. 16. Mcasured oscillograms for a basic two-integrator loop TACO: horizontal axis: $1.5 \mu \mathrm{s} /$ div.; verical axis: $0.1 \mathrm{~V} / \mathrm{div}$.

Basic Two-Integrator Loop TACO: The so-called basic two-integrator loop is obtained by eliminating the OTA's of transconductance $g_{m 3}$ and $g_{m 4}$ from Fig. 5(b). Fig. 16 shows waveforms measured at the nodes of a breadboard prototype of this circuit for $C_{1}=C_{2}=C=330 \mathrm{pF}$ and a particular setting of $g_{m 1}=g_{m 2}=g_{m}$. Fig. 16(a) for unbuffered OTA's while buffered OTA's are used in Fig. 16(b). In these results the OTA's are acting as limiting devices.

Applying the ideal OTA model to the basic two-integrator loop results in a characteristic equation with a pair of imaginary roots. Hence, this ideal model cannot account for the self-starting limit cycle that is illustrated in Fig. 16. This can however be accounted for by using (28) where $g_{m 3}=g_{m 4}=0$ because the corresponding OTA's are not included in the two-integrator loop. As it can be seen from the resulting equation, the effect of $\omega_{z}$ is to make the circuit to be self-starting, the amplitude being stabilized by the OTA intrinsic nonlinearity. The amplitude enlargement and frequency decrease that are observed when using buffered OTA's (Fig. 16b) can be also explained from (28) by observing that an effective decrease in $G_{i}$ forces the roots to move further into the right-half plane, thereby forcing the control mechanism to work harder to stabilize the amplitude.

Externally Controlled TACOs: Previous result suggests the possibility of exploiting OTA parasitics for simple TACO design. The price paid for this is a poor control on the $s$-term of the characteristic equation, what reduces the oscillation frequency and increases the distortion. Much better performance can be obtained by an external control. To illustrate this point we have breadboarded the whole quadrature TACO of Fig. 5(b) and used a diode implementation for the nonlinear resistor of Fig. 9, to control the amplitude. The same tuning conditions for $g_{m 1}$ and $g_{m 2}$ and $C$-values as for Fig. 16(b) were used. However, in contrast to the $14.8 \mathrm{kHz}$ measured for the high-distortion signal of Fig. 16(b), the measured frequency and THD were now $653 \mathrm{kHz}$ and 1.1 percent, respectively. A significant improvement in performance is observed!

To further illustrate the performance of externally controlled TACO's. Fig. 17 shows an oscillogram and its corresponding spectrum for a breadboard prototype of the bandpass TACO of Fig. 6(b). A THD of 0.6 percent was measured for a $303-\mathrm{kHz}$ oscillation.

In order to validate our results for a fully integrated oscillator, a $3-\mu \mathrm{m}$ CMOS double metal (through and thanks to MOSIS) prototype for the quadrature oscillator has
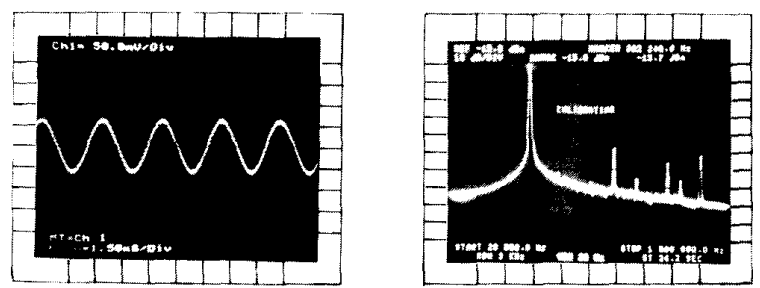

Fig. 17. Measured oscillogram and spectrum for the bandpass TACO of Fig. 6(b) including a nonlinear resistor for amplitude stabilization.

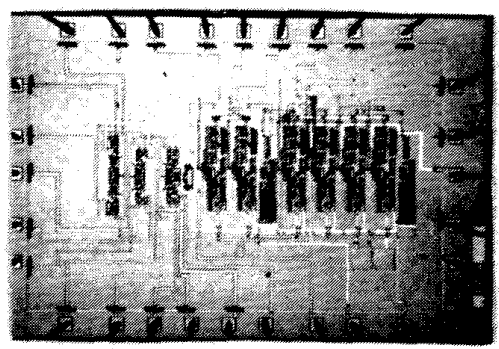

(a)

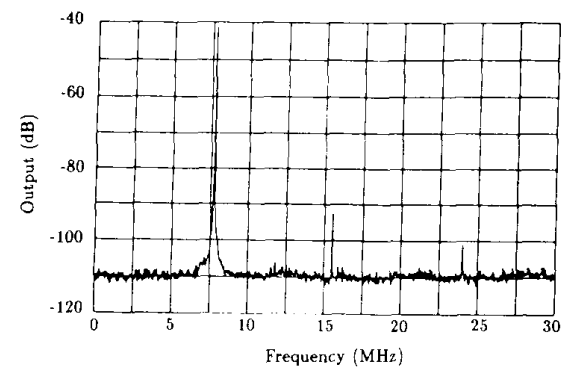

(b)

Fig. 18. Microphotograph of a $3-\mu \mathrm{m}$ double metal CMOS TACO prototype. (b) Measured spectrum for a $7.76 \mathrm{MHz}$ waveform.

been fabricated, the OTA being similar to that in [14] with increased $g_{m}$ and the nonlinear resistor being implemented as a two diodes connection of two MOS transistors. Fig. 18(a) shows a microphotograph of the prototype including the TACO and several buffers for testing. The nominal value for each capacitor was $2 \mathrm{pF}$. Frequencies up to 7.76 $\mathrm{MHz}$ were obtained experimentally. Fig. 18(b) shows the measured spectrum of the $7.76 \mathrm{MHz}$ waveform exhibiting a THD of 0.29 percent. The corresponding peak to peak amplitude was $200 \mathrm{mV}$. It is worth mentioning that these results on the CMOS case are very preliminary. Our interest in including these results here is for illustrating the broad validity of the theoretical framework for TACO design presented in the paper.

AGC Controlled Quadrature-TACO: Fig. 19(a) an OTA-based AGC loop to control the transconductance $g_{m 4}$ of Fig. 5(b). Analysis taking into account that the transconductance of the bipolar OTA is proportional to the bias current [18] gives

$$
g_{m 4}=\frac{1}{2 V_{t}}\left[I_{0}+F\left(k_{0} A-E_{\mathrm{ref}}\right)\right]
$$

where $V_{t}=k T / q$ and $F(\cdot)$ is the nonlinear OTA transconductance defined in Fig. 15. By combining (29) with (27), 


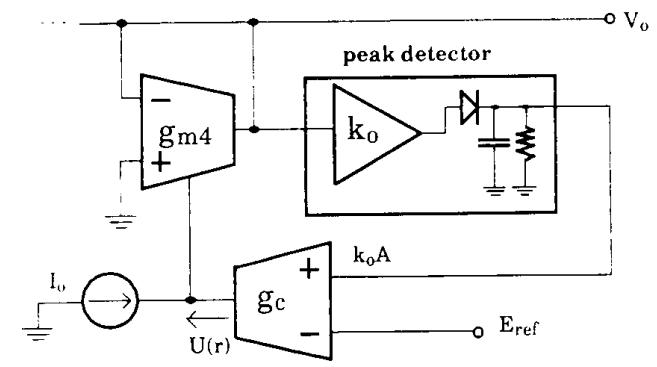

(a)

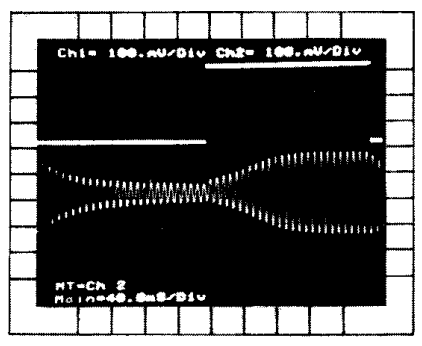

(b)

Fig. 19. (a) AGC loop for amplitude stabilization of a quadrature TACO. (b) Measured steady-state waveform and transient response of the quadrature TACO including an AGC mechanism. Top vertical signal: $E_{\text {ref }} 0.1 \mathrm{~V} /$ div.; bottom vertical: oscillator output, $0.1 \mathrm{~V} /$ div.: Horizontal signal; time, $40 \mu \mathrm{s} / \mathrm{div}$

and assuming $g_{m 3}=0$, we get, for the characteristic equation:

$$
\begin{aligned}
s^{2}-\frac{s}{C} \mid \frac{2 g_{m 1} g_{m 2}}{\omega_{z} C^{2}} & -\frac{I_{0}}{2 V_{t} C}-\frac{4\left(G_{0}+G_{i}\right)}{C} \\
& -\frac{1}{2 V_{t}} F\left(k_{0} A-E_{\mathrm{ref}}\right) \mid+\frac{g_{m 1} g_{m 2}}{C^{2}}=0
\end{aligned}
$$

A stable oscillation with amplitude $A_{0}=E_{\text {ref }} / k_{0}$ can be obtained by choosing the element values to make the sum of the three first terms of the $s$-coefficient of (30) to vanish. This principle has been proven for a number of designs (using discrete bipolar junction transistor (BJT) OTAs) up to a frequency of $200 \mathrm{kHz}$. For all the cases the measured THD was less than 0.27 percent. The oscillogram in Fig. 19(b) illustrates the variation in amplitude under a step change in the value of the reference voltage for a frequency of $196 \mathrm{kHz}$.

Voltage-Controlled Quadrature TACO: The operation of TACO's as wide range VCO's is illustrated using a breadboard prototype of Fig. 5(b), where the amplitude is controlled by a nonlinear resistor. Using (13) and (14b), and taking into account (27), we get the following oscillation frequency and amplitude:

$$
\begin{aligned}
& \omega_{0}=\frac{g_{m 1} g_{m 2}}{C^{2}} \\
& A_{0}=E N_{1}^{-1}\left\{1-\frac{2 g_{m 1} g_{m 2}}{\omega_{2} C^{2} G_{c}}+\frac{4\left(G_{0}+G_{i}\right)}{C G_{c}}-\frac{g_{m 3}-g_{m 4}}{C G_{c}}\right\}
\end{aligned}
$$

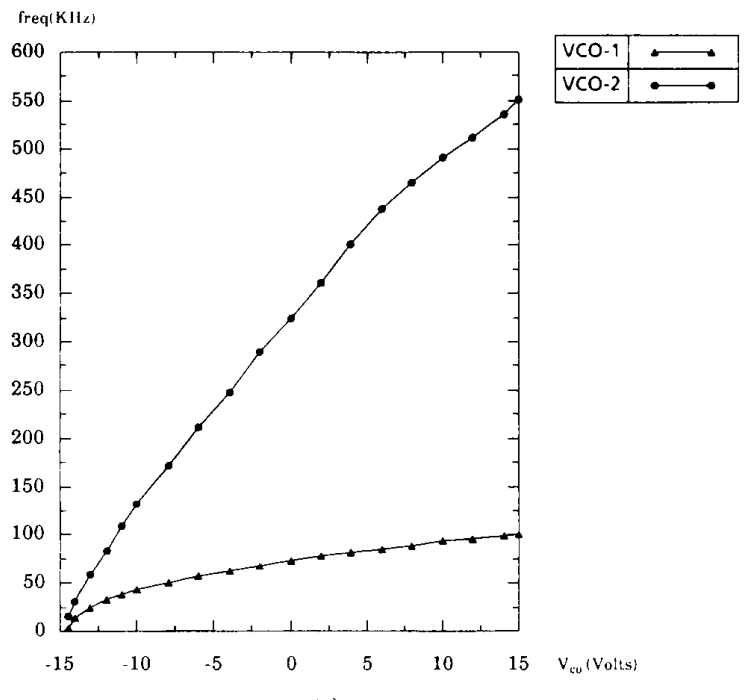

(a)

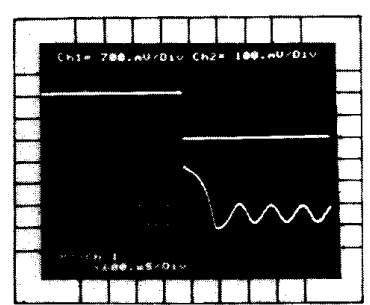

(b)

Fig. 20. (a) Measured frequency versus $V_{c 0}$ for the circuit of Fig. 29. (b) measured oscillogram illustrating the VCO operation. Top vertical signal: $V_{c 0}, 0.7 \mathrm{~V} /$ div.; bottom vertical: oscillator output, $0.1 \mathrm{~V} /$ div.; Horizontal signal: $0.6 \mathrm{~ms} / \mathrm{div}$

where $N_{I}(\cdot)$ is defined by $(15 \mathrm{~b})$ and $G_{c}$ and $E$ are defined in Fig. 9. For VCO operation either $g_{m 1}$ or $g_{m 2}$, or both, have to be tuned. However, since OTA parasitics introduce a coupling between the frequency and the amplitude (see (31)) a more involved tuning strategy is required to guarantee that the amplitude and hence the distortion level of the oscillation would remain unchanged for a variation in either $g_{m 1}$ or $g_{m 2}$. From (31) it is seen that in order to keep the amplitude fixed as $g_{m 1}\left(g_{m 2}\right)$ varies, the following must be fulfilled:

$$
\frac{2 g_{m 1} g_{m 2}}{C^{2} \omega_{z}}-\frac{g_{m 4}-g_{m 3}}{C}=c t e
$$

which means that either $g_{m 3}$ or $g_{m 4}$ must be made to vary to compensate the effect of the $g_{m 1}\left(g_{m 2}\right)$ changes.

A breadboard prototype including a tuning strategy based on this formula has been devised. Fig. 20(a) shows the measured oscillation frequency versus the control voltage $V_{c 0}$ for two different cases, namely making both $g_{m 1}$ and $g_{m 2}$ to vary (this is the case labelled VCO-2) and maintaining constant the $g_{m 2}$ value (VCO-1). For all the measured frequencies, the amplitude was set to a constant value of $100 \mathrm{mV}$. Fig. 20(b) illustrates the VCO operation under a step change in the voltage control signal. 


\section{Conclusions}

A unified theoretical approach to design sinusoidal oscillators using OTA's as the active device has been presented. The potential advantages of these oscillators as compared to conventional opamp-based designs have been discussed. For illustration purposes, four different structures have been reported and analyzed by using both linear and nonlinear models. A practical OTA macromodel has been developed and applied to the design of practical AGC-controlled oscillators and wide-range VCO's. The experimental results that have been obtained have an excellent agreement with the theoretical results and show good potential for VCO high-frequency applications where opamp circuits are not applicable. It was shown that using CMOS OTA's [27], [28] together with the TACO structures reported herein can be a good solution for the realization and high-frequency monolithic sinusoidal oscillators and VCO's.

\section{REFERENCES}

[1] K. K. Clarke and D. T. Hess, Communication Circuits: Analysis and Design. Reading, MA: Addison-Wesley, 1978.

[2] Y. Tsividis and P. Antognetti, Design of MOS VLSI Circuits for Telecommunications. Englewood Cliffs, NJ: Prentice-Hall, 1985.

[3] V. Prem-Pyara, S. C. Dutta-Roy, and S C. Jamuar: "Identification and design of single-amplifier single-resistor controlled oscillators,"

[4] B. B. Battacharyya and M. Tavakoli-Darkani, "A unified approach to the realization of canonic $R C$-active single as well as variable frequency oscillator using operational amplifiers," J. Franklin Inst. vol. 317, pp. 413-439, June 1984.

[5] N. Boutin, "On the identification and Design of single-amplifier single-resistance controlled oscillators," IEEE Trans. Circuits Syst., vol. CAS-31, pp. 1046-1048, Dec. 1984

[6] M. Tavakoli-Darkani and B. B. Battacharyya, "Generation and design of canonic grounded-capacitor variable-frequency $R C$-active oscillators," Proc. Inst. Elect. Eng., vol. 132, pt. G, pp. 153-160, Aug. 1985 .

[7] J. L. Huertas, A. Rodríguez-Vázquez, and B. Pérez-Verdú, "Highfrequency design of sinusoidal oscillators realized with operational amplifiers," Inst. Elect. Eng., vol. 131, pt. G, pp. 137-140, Aug.
1984 .

[8] A. Budak and K. Nay, "Operational amplifier circuits for the Wien-bridge Oscillator," IEEE Trans. Circuits Syst., vol. CAS-28, pp. 930-934, Sept. 1981.

[9] A. Rodríquez-Vảzquez, J. L. Huertas, and B. Pérez-Verdú: “Highfrequency design of the Wien-bridge oscillator using composite amplifiers," IEEE Trans. Circuits Syst., vol. CAS-34, pp. 441-443, amplifiers,"
Apr. 1987.

[10] M. Hribsek and R. W. Newcomb: "VCO controlled by one variable resistor," IEEE Trans. Circuits Syst., vol. CAS-23, pp. 166-169, Mar. 1976.

[11] K. D. Peterson, A. Nedungadi, and R. L. Geiger: "Amplifier design considerations for high frequency monolithic filters," in Proc. 1987 European Conf. on Circuit Theory and Design, pp. 321-326, Sept. 1987.

[12] F. Krummenacher and N. Joel, "A 4-MHz CMOS continuous-time filter with on-chip automatic tuning," IEEE J. Solid-State Circuits, vol. SC-23, pp. 742-749, June 1988 .

[13] C. S. Park and R. Schaumann, "Design of a 4-MHz analog integrated CMOS transconductance- $C$ bandpass filter," IEEE $J$ Solid-State Circuits, vol. SC-23, pp. 987-996, Aug. 1988

[14] A. Nedungadi and R. L. Geiger, "High-frequency voltage Controlled Continuous time low-pass filter using linearized CMOS integrators," Electron. Lett., vol. 22, pp. 729-731, June 1986.

[15] A. Buonomo, C. Di Bello, and O. Greco: "On the existence and uniqueness of stable quasi-sinusoidal oscillations," Int. J. Circuit Theory Appl., vol. 13, pp. 327-335, 1985.

[16] A. Gelb and W. Vander Velde, Multiple-Input Describing Function and Nonlinear System Design. New York: McGraw-Hill, 1968.

[17] A. R. Bergen, L. O. Chua, A. I. Mees, and E. W. Szeto: "Error bounds for general describing function problems," IEEE Trans. Circuits Syst., vol. CAS-29, pp. 345-354, June 1982.

[18] R. L. Geiger and E. Sánchez-Sirencio, "Active Filter Design Using OTAs: A tutorial," IEEE Circuits and Devices Magazine, vol. 1, pp. 20-32, March 1985.
[19] E. Sáchez-Sinencio, R. L. Geiger, and H. Nevarez-Lozano: "Generation of Continuous-Time Two Integrator Loop OTA Filter Structure." IEEE Trans. Circuits and Systems, vol. CAS-35, pp. 936-946, August 1988 .

[20] M. Bialko and R. W. Newcomb, "Generation of All Finite Linear Circuits Using the Integrated DVCCS". IEEE Trans. Circuits Theory, vol. CT-18, pp. 733-736, Nov. 1971.

[21] B. Linares-Barranco "Design of quasi-sinusoidal oscillators using OTA's," Master's thesis, Univ. of Seville, Spain, Sept. 1987 (in Spanish).

[22] R. Wojtyna and A. Borys, "Contribution to the linear theory of frequency stability of $R C$ oscillators," IEEE Trans. Circuits Syst. vol. CAS-33, pp. 418-424, Apr. 1986.

[23] L. O. Chua C. A. Desoer, and E. S. Kuh, Linear and Nonlinear Circuits. New York: McGraw-Hill, 1987

[24] P. E. Allen and D. R. Holberg, CMOS Analog Circuit Design. New York: Holt, Rinehart, Winston, 1987

[25] H. Nevárez-Lozano, J. A. Hill, and E. Sánchez-Sinencio, "Frequency limitations of continuous-time OTA-C filters," in Proc. IEEE Int. Symp. on Circuits and Systems, vol. 3, pp. 2169-2172, June 1988.

[26] J. Hoyle and E. Sánchez-Sinencio, "Sinusoidal quadrature OTA oscillators," Proc. 27th Midwest Symp. on Circuits and Systems, pp. 59-62, Aug. 1984

[27] . A. Nedungadi and T. R. Viswanathan, "Design of linear CMOS transconductance elements," IEEE Trans. Circuits Syst., vol. CAS31 , pp. $891-894$, Oct. 1984 .

[28] R. R. Torrance, T. R. Viswanathan, and J. V. Hanson, "CMOS voltage to current transducers (VCT)," IEEE Trans. Circuits Syst. vol. CAS-32, pp. 1097-1104, Nov. 1985

[29] W. B. Mikhael and S. Tu, "Continuous and switched-capacitor multiphase oscillators," IEEE Trans. Circuits Syst., vol. CAS-31, pp. 280-293, Mar. 1984.

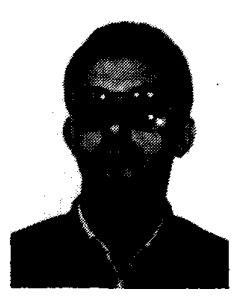

Angel Rodríguez-Vázquez (M'80) received the Licenciado en Fisica degree in 1977, and the Doctor en Ciencias Físicas degree in 1983, both from the University of Seville, Spain.

Since October 1978 he has been with the Departamento de Electrónica y Electromagnetismo at the University of Seville, where is currently employed as an Associate Professor. His research interest lies in the fields of analog/digital integrated circuit design, analog neural networks and modeling of nonlinear phenomena in integrated circuits.

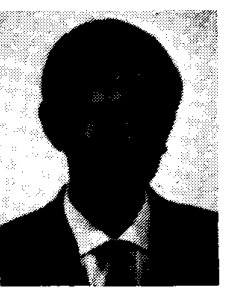

José L. Huertas (M'74) received the Licenciado en Fisica degree in 1969, and the Doctor en Ciencias Físicas degree in 1973, both from the University of Seville, Spain.

From October 1970 to September 1971 he was with the Philips International Institute, Eindhoven, The Netherlands, as a post-graduate student. Since October 1971 he has been with the Departamento de Electrónica y Electromagnetism at the University of Seville, where is currently employed as Professor. His research interest lies in the fields of multivalued logic, sequential machines, analog circuit design and nonlinear network analysis and synthesis. 


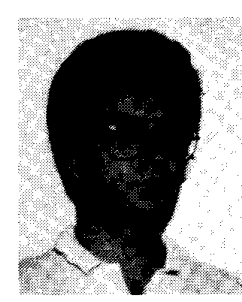

Bernabé Linares-Barranco received the B.Sc. degree in electronic physics in 1986 and the M.S degree in microelectronics in 1987 from the University of Seville, Seville, Spain. Currently he working toward the Ph.D. degree in electrical engineering at the Texas A\&M University, College Station.

His research interests are in the area of nonlinear analog microelectronic design and neural networks.

\section{E}

Edgar Sánchez-Sinencio (S'72-M'74-SM'83) received the M.S.E.E. degree from Stanford University, CA and the $\mathrm{Ph} . \mathrm{D}$. degree from the University of Illinois at Champaign-Urbana, in 1970, and 1973, respectively.

In 1974, he held an industrial Post-Doctoral position with the Centra Research Laboratories, Nippon Electric Company, Ltd., Kawasaki, Japan.

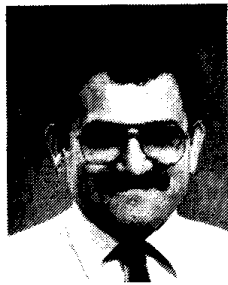

From 1976 to 1983 , he was the Head of the Department of Electronics at the Instituto $\mathrm{Na}$ cional de Astrofísica, Optica y Electrónica, Puebla, Mexico. He was a Visiting Professor at Texas A\&M University during 1979-1980 and 1983-1984, where he is currently a Professor.

His present interests are in the area of solid-state circuits, including CMOS neural network implementations, and computer-aided circuit design

He was the General Chairman of the 1983 26th Midwest Symposium on Circuits and Systems. He was an Associate Editor of News and Events for the IEEE Circuits and Devices Magazine from 1985 to 1988 . He was an Associate Editor for the IEEE TRANSACTIONS ON CIRCUITS AND SYSTEMS from 1985 to 1987. $\mathrm{He}$ is currently the President-Elect of the IEEE Circuits and Systems Technical Committee on Neural Systems and Applications. He is the co-author of the book Switched-Capacitor Circuits (Van Nostrand-Reinhold, 1984). 